\title{
Texture Analysis by Neutron Diffraction Using a Linear Position Sensitive Detector
}

\author{
G. WILL, W. SCHÄFER and P. MERZ
}

Mineralogical Institute, University of Bonn, Poppelsdorfer SchloB, D-5300 Bonn, West Germany

(Received 17 May, 1989)

Neutron diffraction in connection with a position-sensitive detector is a most powerful technique in texture analysis comparable in time with conventional X-ray laboratory technique. Neutrons measure the global texture of the sample allowing volumes up to several $\mathrm{cm}^{3}$. By using position-sensitive detectors and applying the mathematical procedures of profile analysis multiphase and low symmetry materials can be investigated without serious difficulties. Neutron diffraction experiments operating in transmission record complete diffraction profiles; overlapping lines are unscrambled by profile analysis. Technical and physical specifications of the dedicated detector JULIOS, installed on the texture diffractometer of Bonn University, are given. A hematite ore has been studied by this technique and the results are given.

KEY WORDS: Position sensitive solid state detector, peak profile analysis, pole figure measurement, Hematite, Biotite.

\section{INTRODUCTION}

Texture analysis by X-ray diffraction is a common and well established technique, and especially in metallurgical applications it is widely used. Texture of a polycrystalline sample means the orientation distribution of the many crystallites with respect to a reference system on the sample (for example the rolling transverse and normal direction). The classical procedure is to keep the detector at a fixed position $2 \Theta$ of a possible Bragg reflection hkl and 
to turn the sample in space on an Eulerian cradle stepwise through the whole space about two angles $\varphi$ and $\chi$ (Figure 1). There are different modes of operation: equal step scanning or equal area scanning. We are using equal area scanning with generally 621 angular positions $\varphi$ and $\chi$. The scan pattern for equal area scanning is shown in Figure 2. Finer steps can be (and are) used by us if required.

Neutron diffraction as a source for texture analysis is a comparatively new method with its first attempts dating back to 1953 and 1968 (Brockhouse, 1953; Bunge and Tobisch, 1968). It was only very recently that considerable progress has been made in studying by neutron diffraction also complicated samples consisting of several phases of even low symmetry, to obtain pole figures and to calculate orientation distributions (ODF's).

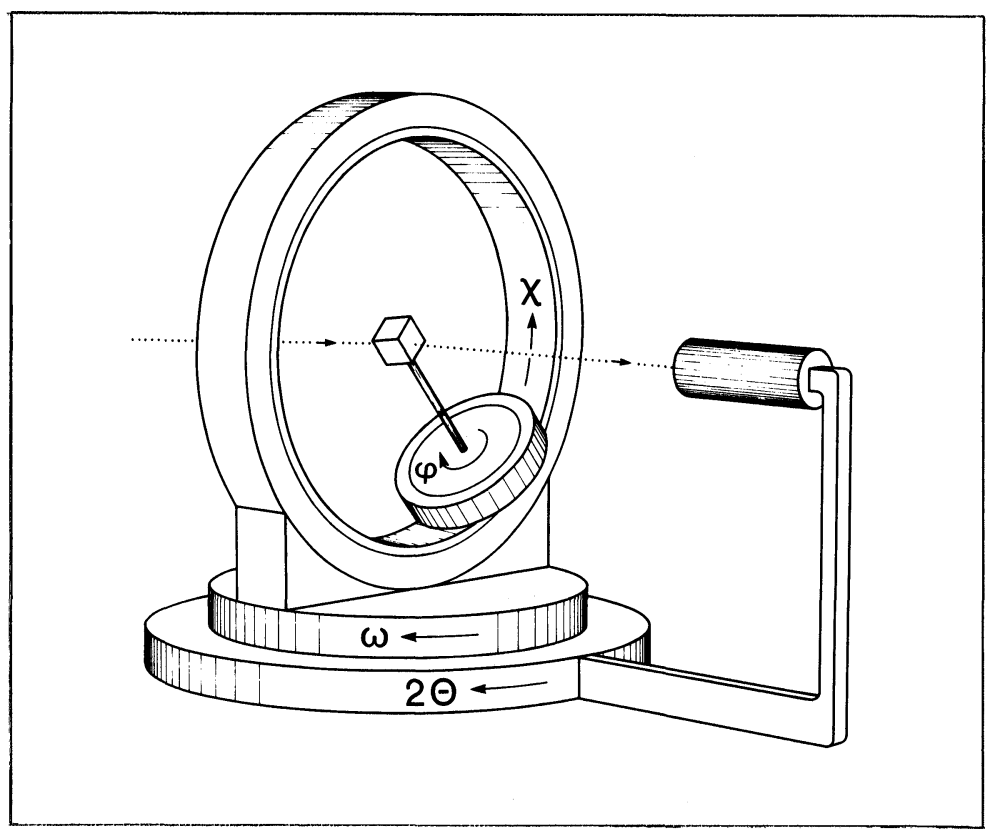

Figure 1 Eulerian cradle with rotational possibilities around $\varphi, \chi$ and $\omega$, sitting on a detector base. Shown is the conventional set-up with a single detector at scattering angle $2 \Theta_{0}$. This detector has meanwhile been replaced by a position sensitive detector as shown in Figure 4. 


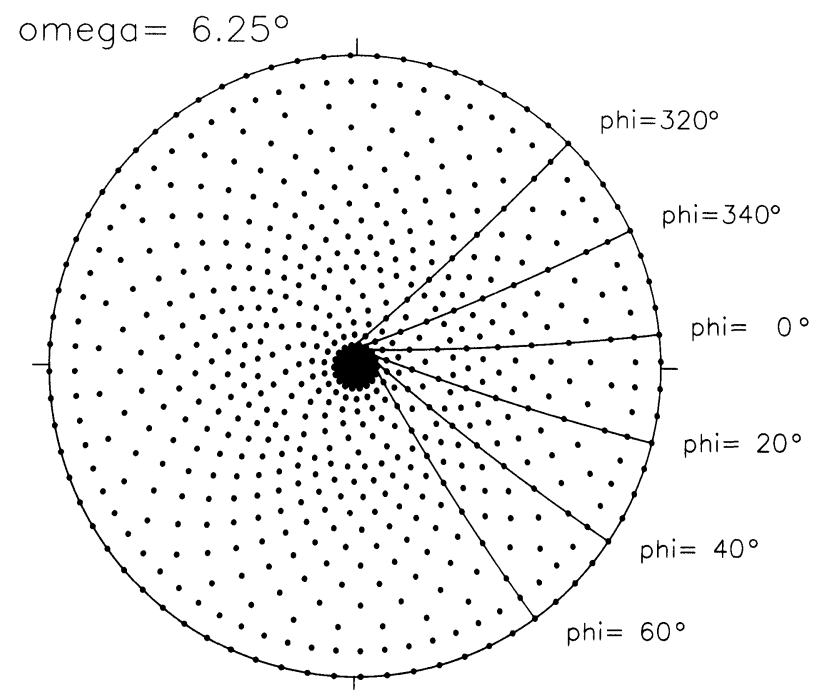

Figure 2. Pole figure scanning pattern as it is used by us with the position-sensitive detector JULIOS. There is a blind area at the center for the data points measured away from the center angle $2 \Theta_{0}$. The size of the blind area increases from zero to maximum $\omega=6.25^{\circ}$ at the outer end of our detector. The situation is shown in the picture.

The advantages and draw backs of both methods, X-rays and neutrons, are obvious: texture analysis is a statistical description and therefore requires a large number of crystallites in order to get a meaningful sampling. It is generrally accepted that 10,000 grains or crystallites are a good figure. The well known properties of X-rays: large absorption in the sample and a small beam diameter limit its use to a) small samples, and b) fine grained samples with grains in the order of 10 to $20 \mu$. These limitations lead to study only the surface of a sample because the X-rays penetrate the sample just a few microns. Second, there are limitations in sample rotation and therefore only incomplete pole figures can be obtained. (These drawbacks are sometimes overcome by clever techniques, however, in this connection only the principle features are of interest). X-rays measure the "local" texture at the sample surface.

On the other hand neutron beams are large up to $100 \times 50 \mathrm{~mm}^{2}$, neutrons are in general little absorbed in the material and as a 
consequence we can measure large samples several $\mathrm{cm}$ in diameter, we can study samples with coarse grains ranging up to millimetres, we can penetrate and therefore explore the whole sample, we can measure in transmission and therefore record and calculate complete pole figures. Neutrons measure the "global" texture. (See Will, Höfler and Schäfer, 1986; Schäfer, Höfler and Will, 1988).

Because of significant differences in the scattering powers of the elements for X-rays and neutrons both methods are complementary in many cases and in combination they are therefore an excellent and powerful technique. This was demonstrated for example in an earlier study on calcite, $\mathrm{CaCO}_{3}$, (Wenk, Kern, Schäfer and Will, 1984) where some reflections like 00.6 are too weak in the $X$-ray diagram, however very strong in the neutron diagram. For 11.0 and 20.2 the reverse is true.

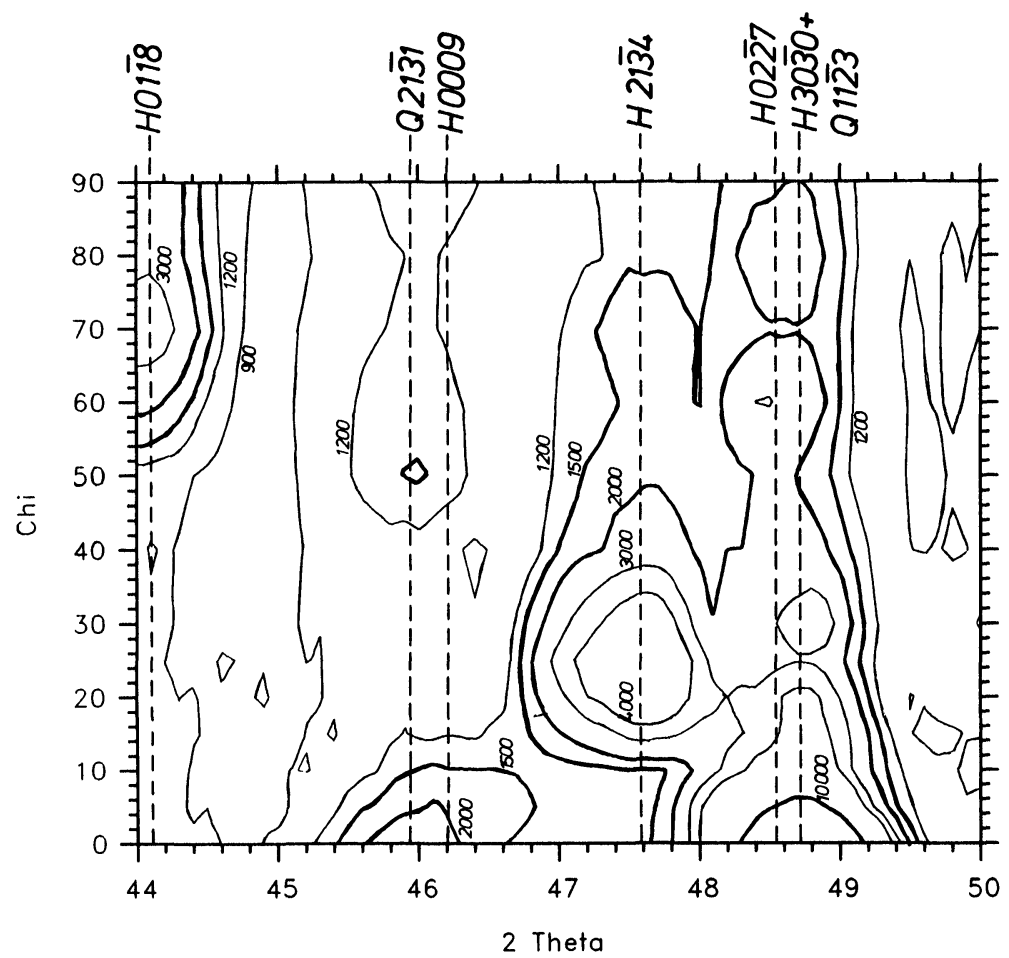

Figure 3 A series of $\Theta-2 \Theta$ scans of a hematite ore plotted for $\chi$-angles varying from $\chi=0^{\circ}$ to $\chi=90^{\circ}$. The calculated $2 \Theta$-angles of hematite and quartz are indicated by vertical lines. 
In multiphase samples, like rocks or ores, often containing low symmetry mineral crystals, but also in modern ceramic materials the diffraction patterns contain a large number of reflections which in general are highly overlapped. For a successful texture analysis of such samples two requirements have to be fulfilled:

1. We must measure an extended portion of the diffraction diagram, e.g. we must measure the whole peak or a group of peaks and not just stationary at the peak maximum. Figure 3 depicts a series of $\Theta-2 \Theta$ scans over $6^{\circ}$ in $2 \Theta$ of a hematite ore for varying $\chi$-angles with the $\chi$-circle stationary at each measurement. The region around $2 \Theta=46^{\circ}$ demonstrates very convincingly that scanning in a stationary mode may give wrong results. The hematite reflection 00.9 and the quartz reflection 21.1 are very close to each other and so strongly overlapped that peak intensities cannot be deduced from one angular position.

2. We must separate or deconvolute overlapping peaks.

The first requirement is fulfilled by using position sensitive or area-detectors. The second one can be done by mathematical procedures of profile analysis.

\section{POSITION SENSITIVE DETECTORS}

The first linear position sensitive detector (PSD) commercially available was the so-called "BANANA" and it was used in texture analysis with neutron diffraction at the ILL, Grenoble, France (Bunge, Wenk and Pannetier, 1982; Wenk, Bunge, Jansen and Pannetier, 1986). The BANANA is a $\mathrm{BF}_{3}$-gas detector constructed as a multi-wire system. Spatial separation of the wires determines the angular resolution and this is about $0.2^{\circ}$ in $2 \Theta$ for the early BANANA. The detector is bent and therefore constructed for a fixed distance from the sample. Today, the $\mathrm{BF}_{3}$-gas technology is considered obsolete and widely replaced by inert $\mathrm{He}-3$ gas systems because of more favourable technical and physical conditions with respect to sensitivity and spatial resolution.

In a number of places, especially in Germany and Japan developments have taken place to built position sensitive detectors on solid state scintillator basis. One such detector, called JULIOS, 
has been developed in Jülich (Schäfer, Jansen, Elf and Will, 1984) and is commercially available. The detection medium is a $\mathrm{Li}^{6}$ containing glass plate coupled to a set of 24 linearly arranged photomultipliers.

The position of an event is determined in analogy to the so-called Anger-technique by an electronic processing of different anode currents coming from 3 photomultipliers (Figure 4). By a small airgap and with the refraction index of the glass plate there are always not more than three multipliers illuminated. Technical and physical specifications of JULIOS are summarized in Table 1 . The solid state detector is characterized by a high detection efficiency of about $80 \%$ for $1.8 \AA$ neutrons. The planar detector form allows to operate at variable distances to the sample in order to optimize for

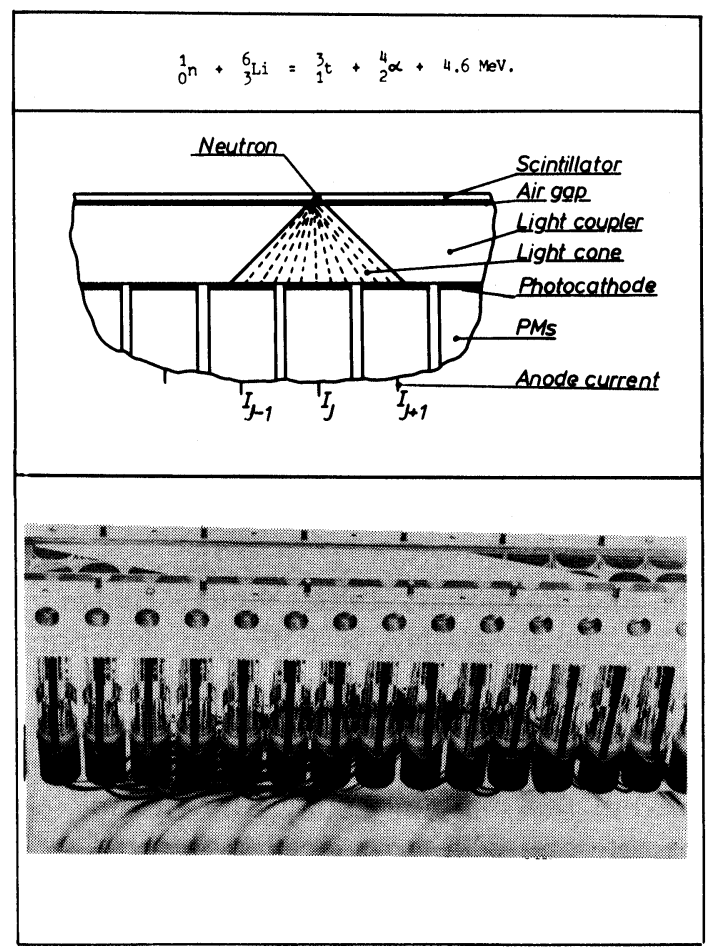

Figure 4 Schematic representation (above) and practical realization (photo below) of the linear scintillation detector JULIOS. 
Table 1 Detector specifications

\begin{tabular}{ll}
\hline $\begin{array}{l}\text { Scintillator material } \\
\text { Detector form }\end{array}$ & $\begin{array}{l}\mathrm{Li}^{6} \text {-glass } \\
\text { planar } \\
\text { Effective length }\end{array}$ \\
$\begin{array}{l}682 \mathrm{~mm} \\
\text { Covered } 2 \Theta\end{array}$ & \\
$100 \mathrm{~cm}$ distance to sample: & $36^{\circ}$ \\
$150 \mathrm{~cm}$ distance to sample: & $25^{\circ}$ \\
Detection efficiency & $65 \%$ \\
$\quad$ for $1.0 \AA$ neutrons: & $85 \%$ \\
for $2.0 \AA$ neutrons: & $2.5 \mathrm{~mm}$ \\
Linear spatial resolution: & $0.1^{\circ}$ \\
Angular resolution: & $0.025^{\circ}$ \\
Channel distance: & $10^{-3}$ \\
Resolution $\Delta \mathrm{d} / \mathrm{d}$ for $1.2 \AA:$ & 40 \\
Number of channels per deg in $2 \Theta:$ & 990 \\
Total number of channels: & $1 \mathrm{MHz}$ \\
Count rate capability: & \\
\hline
\end{tabular}

angular resolution. One specific aspect in using PSDs for pole figure measurements is the so called "blind area" (Bunge $1982 \mathrm{a}$ and b). When using PSDs the $\chi$-circle of the Eulerian cradle coincides with the bisectrix of the incident and the reflected beam only for reflections in the center of the detector. The scan figures of reflections registered at the outer parts of the detector are characterized by a blind area which increases with the distance of the position of the reflection to the position of the detector centre. The blind area has to be covered by additional measurements either in additional $\varphi$-, $\chi$-orientations or in complete additional scanning pattern for a different sample adjustment on the Eulerian cradle. The economic effect of reducing the measuring time by using a PSD in texture analysis is not directly proportional to the detector length. In the case of JULIOS with an effective length of $682 \mathrm{~mm}$ and covering about $25^{\circ}$ in $2 \Theta$, the blind area is filled by one additional measurement for each reflection with $\chi=90^{\circ}$ and $\varphi=0^{\circ}$.

\section{PEAK SEPARATION BY PROFILE ANALYSIS}

The crucial point in data analysis of multiphase and/or low symmetry materials is the separation of overlapping peaks. This can be done by profile analysis. In the authors' laboratory powerful 
programs have been developed (Jansen, Schäfer and Will, 1986 and 1988). In contrast to X-ray diffraction, where the profiles are complicated and vary with diffraction angle (Will, Parrish and Huang 1983), the conditions in neutron diffraction are much simpler. The peak shape of a neutron diffraction reflection can be well described by a Gaussian function. Variation with scattering angle effects only the half width, which is determined by using a standard sample, in our case silicon.

We spend about 1 to 4 minutes at each sample position, and the individual diagram, as shown in Figure 5 for $\varphi=20^{\circ}, \chi=10^{\circ}$ ) is of rather poor counting statistics. A further serious handicap is the background. For analysing the measurement we have developed the following procedure operating in 4 successive steps:

1) A sum-diagram is generated by adding up all the 621 individual $\varphi-\chi$ diffraction patterns.

2) The background of the sum-diagram is determined by polygons, polynoms or splines. This yields a background-shape-function

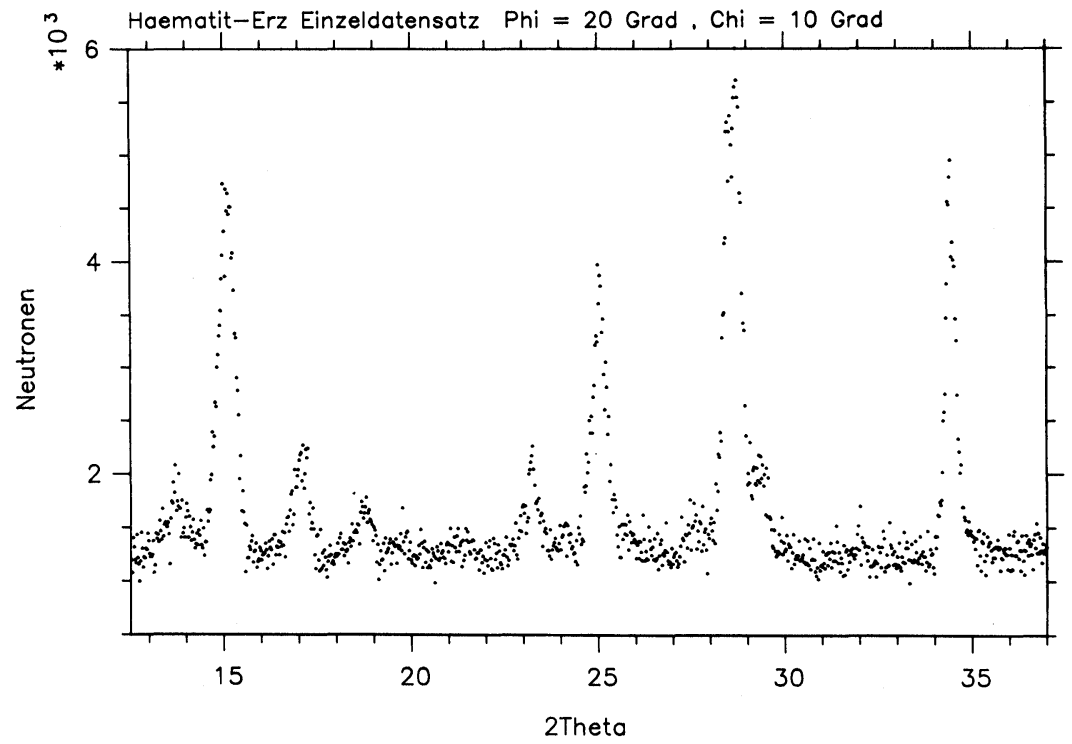

Figure 5 Individual diffraction diagram with $\varphi=20^{\circ}$ and $\chi=10^{\circ}$. The time spent was 3 minutes. 
which is used later in step 4 for background adjustment of the individual diagrams.

3) $2 \Theta$-positions, halfwidths (FWHM) and intensities of the sumdiagram reflections are determined by profile fitting and deconvolution of overlapping peaks using the programs PROFAN or FULFIT (Jansen, Schäfer and Will, 1988).

Profile fitting in PROFAN is started by dividing the measured diffraction diagram into several smaller sections. The profile analysis is then done interactively in front of a screen with the help of a cursor. The cursor is placed on expected peak positions, which can be found either mathematically through the second derivative, or by calculating theoretical powder diagrams using e.g. POWLS (Will, 1974; Will, Jansen \& Schäfer, 1983). In texture analysis we can assume that the mineralogical or metallurgical composition of the sample with respect to the phases is known. The resulting $2 \Theta$ positions and FWHM values of all fitted reflections are used as input-parameters for the intensity analysis of the individual $\varphi-\chi$ diffraction patterns. Examples for a profile analysed pattern are given in Figures 6 and 7.

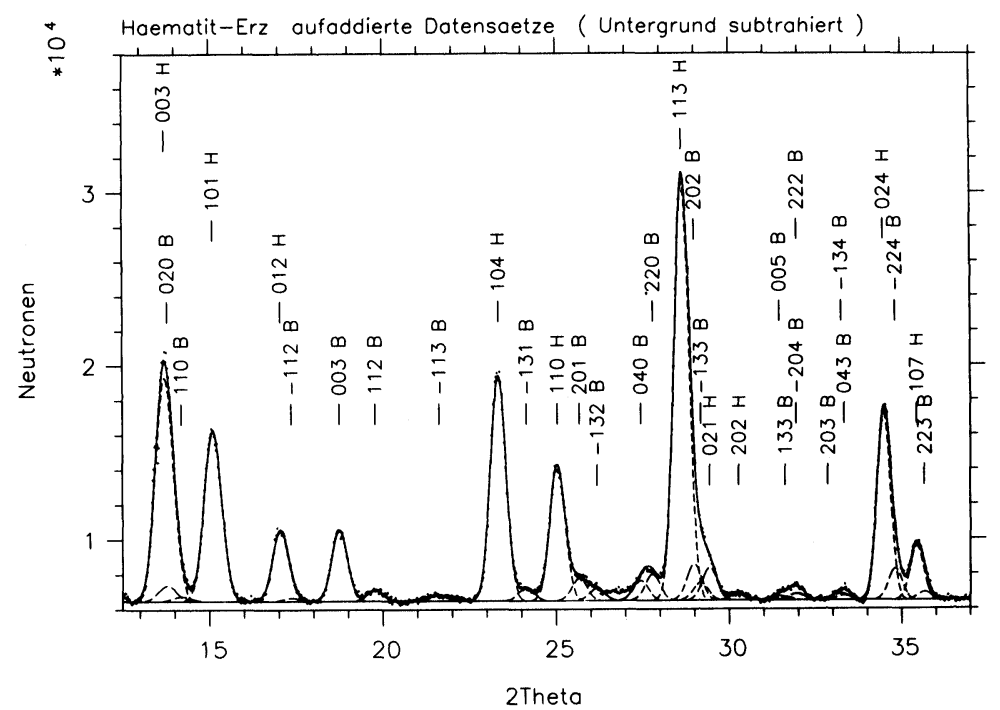

Figure 6 Sum-diagram generated by adding up 621 individual diagrams like that shown in Figure 5. Included is the result of the profile analysis. The indices refer to the two phases, $\mathrm{H}=$ hematite, $\mathrm{B}=$ biotite. 


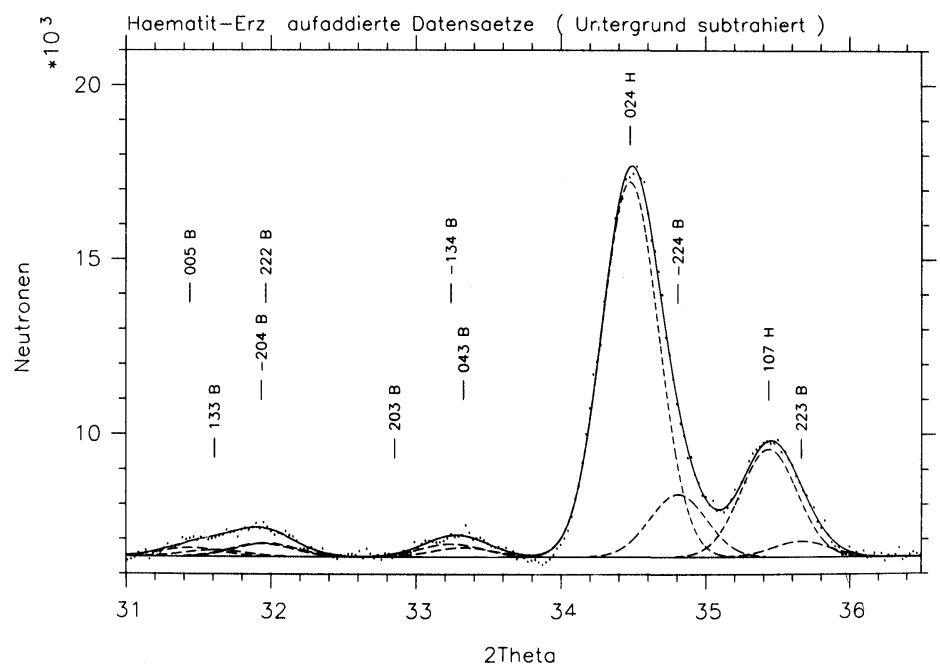

Figure 7 Representative sections of the sum-diagram (\#6 and \#7 of Table 2) showing the result of the profile fitting.

The quality of the fitting is given by a $R$-profile factor:

$$
R_{\mathrm{PF}}=\sqrt{\frac{\sum w_{i}\left(y_{i}^{\text {obs }}-y_{i}^{\text {calc }}\right)^{2}}{\sum w_{i}\left(y_{i}^{\text {obs }}\right)^{2}}}
$$

$y^{\text {obs: }}$ observed intensity, $y^{\text {calc: }}$ calculated intensity, $w$ : weighting factor.

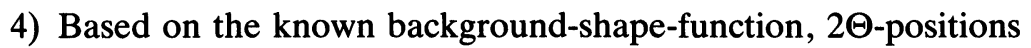
and FWHM values, the orientation dependent intensities of the individual patterns are determined by applying LSQ routines.

\section{POLE FIGURE MEASUREMENTS ON HEMATITE ORE}

The procedure mentioned before has been applied on the texture analysis of a hematite ore using the neutron texture diffractometer 
Table 2 Results of the profile analysis of the sum-diagram subdivided into seven sections

\begin{tabular}{|c|c|c|c|c|c|}
\hline HKL & $2 \theta$ & FWHM & INT. & $R_{\text {exp }}[\%]$ & $R[\%]$ \\
\hline $003 \mathrm{H}$ & 13.71 & 0.6 & 82407 & \multirow{4}{*}{0.93} & \multirow{4}{*}{3.36} \\
\hline 020B & 13.79 & 0.6 & 5841 & & \\
\hline 110B & 14.20 & 0.6 & 1732 & & \\
\hline $101 \mathrm{H}$ & 15.11 & 0.59 & 61279 & & \\
\hline $102 \mathrm{H}$ & 17.04 & 0.54 & 23212 & \multirow{4}{*}{1.13} & \multirow{4}{*}{1.61} \\
\hline$-112 B$ & 17.37 & 0.54 & 1092 & & \\
\hline 003B & 18.75 & 0.53 & 23010 & & \\
\hline 112B & 19.78 & 0.52 & 3719 & & \\
\hline$-113 B$ & 21.62 & 0.52 & 3229 & 1.20 & 1.43 \\
\hline $104 \mathrm{H}$ & 23.33 & 0.52 & 71936 & \multirow{5}{*}{1.00} & \multirow{5}{*}{2.27} \\
\hline$-131 \mathrm{~B}$ & 24.14 & 0.52 & 4252 & & \\
\hline $110 \mathrm{H}$ & 25.04 & 0.52 & 42765 & & \\
\hline 201B & 25.67 & 0.51 & 6981 & & \\
\hline$-132 \mathrm{~B}$ & 26.18 & 0.51 & 3885 & & \\
\hline 040B & 27.34 & 0.51 & 6173 & \multirow{6}{*}{0.96} & \multirow{6}{*}{2.82} \\
\hline 220B & 27.79 & 0.51 & 8319 & & \\
\hline $113 \mathrm{H}$ & 28.63 & 0.51 & 129438 & & \\
\hline 202B & 28.99 & 0.50 & 11155 & & \\
\hline$-133 \mathrm{~B}$ & 29.18 & 0.50 & 5507 & & \\
\hline $201 \mathrm{H}$ & 29.43 & 0.50 & 10021 & & \\
\hline 005B & 31.33 & 0.50 & 1353 & \multirow{7}{*}{1.18} & \multirow{7}{*}{1.42} \\
\hline 133B & 31.61 & 0.50 & 1026 & & \\
\hline$-204 B$ & 31.94 & 0.49 & 2017 & & \\
\hline 222B & 31.97 & 0.49 & 2061 & & \\
\hline 203B & 32.85 & 0.49 & 44 & & \\
\hline$-134 \mathrm{~B}$ & 33.24 & 0.48 & 1968 & & \\
\hline 043B & 33.33 & 0.48 & 1420 & & \\
\hline $204 \mathrm{H}$ & 34.48 & 0.46 & 52569 & \multirow[t]{4}{*}{0.99} & \multirow[t]{4}{*}{1.70} \\
\hline$-224 B$ & 34.81 & 0.46 & 8831 & & \\
\hline $107 \mathrm{H}$ & 35.44 & 0.46 & 15182 & & \\
\hline 223B & 35.66 & 0.46 & 2238 & & \\
\hline
\end{tabular}

of Bonn University at the FRJ-2 in the KFA Jülich, equipped with the PSD JULIOS. The wavelength used was $1.202 \AA$. The detector covered a $2 \Theta$-region from $12.5^{\circ}-37.5^{\circ}$. The sample investigated consisted of two phases-hematite and biotite (Fig. 6). The measurement yielded a total of 31 reflections, of which 15 were used for pole figure representations. Some are shown in Figure 8. 


\section{Haematit-Erz}

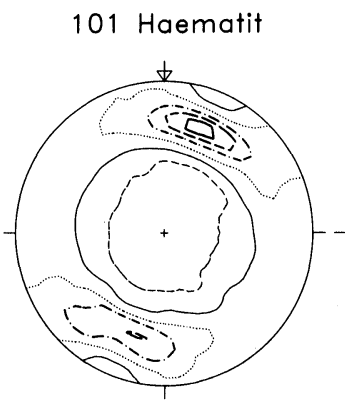
012 Haematit
003 Haematit
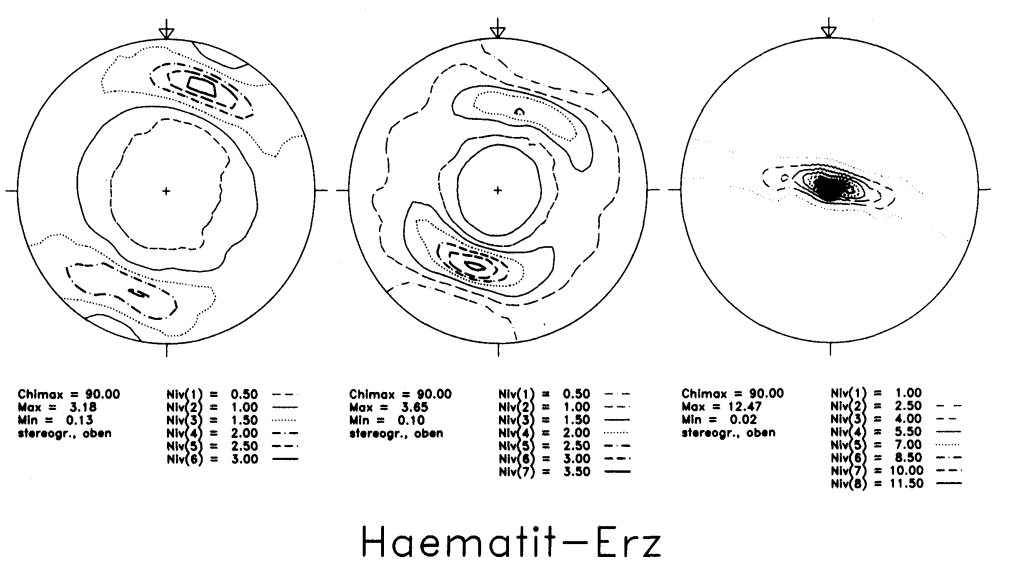

104 Haematit

110 Haematit

113 Haematit
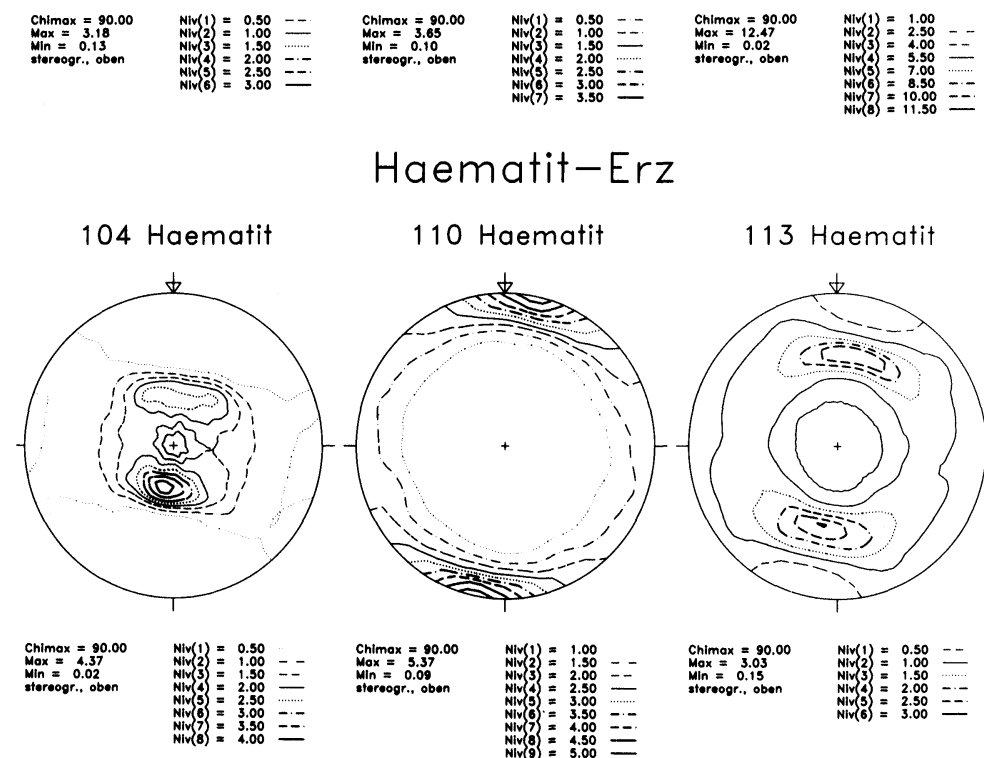

粮:
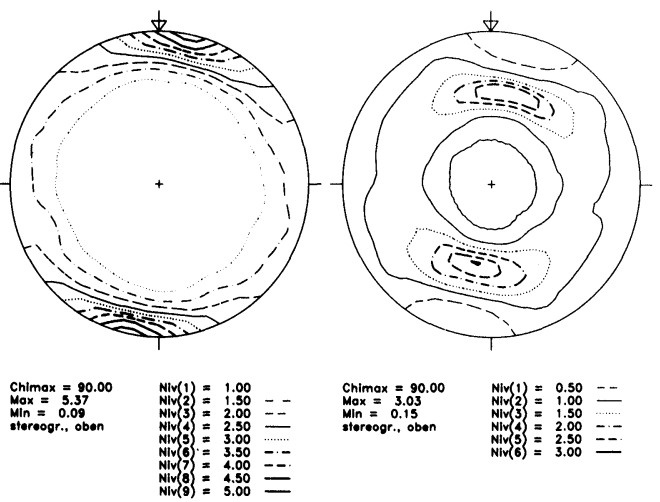

Figure 8 Representative pole figures calculated from the integrated intensities after profile analysis of all 621 individual diagrams. A total of 31 such pole figures were measured, 15 of them were of good quality.

\section{References}

Brockhouse, B. N. (1953). The initial magnetization of nickel under tension. Canad. J. of Physics 31, 339-355.

Bunge, H. J. (1982a). Texture Analysis in Materials Science-Mathematical Methods, London; Butterworths 
Bunge, H. J. (1982b). Experimental Techniques. In: Quantitative Texture Analysis (ed. H. J. Bunge, C. Esling), 85-128. Oberursel; DGM Informationsgesellschaft Verlag.

Bunge, H. J. and Tobisch, J. (1968). Bestimmung der Walztextur des Kupfers mit Hilfe der Neutronenbeugung. Z. Metallkde. 59, 471-475.

Bunge, H. J., Wenk, H. R. and Pannetier, J. (1982). Neutron Diffraction Texture Analysis using a $2 \Theta$-Position Sensitive Detector. Textures and Microstructures 5, 153-170.

Jansen, E., Schäfer, W. \& Will, G. (1986). Application of profile analysis methods in texture measurements using position-sensitive detectors. In: Experimental Techniques of Texture Analysis (ed. H. J. Bunge), 229-240. Oberursel; DGM Informationsgesellschaft Verlag.

Jansen, E., Schäfer, W. \& Will, G. (1988). Profile Fitting and the Two-Stage Method in Neutron Powder Diffractometry for Structure and Texture Analysis J. Appl. Cryst. 21, 228-239.

Schäfer, W., Höfler, S. \& Will, G. (1988). Applications of Neutron Diffraction Pole Figure Measurements on Polycrystalline and Monocrystalline Metallic Samples. Examples from the Four-Circle Neutron Diffractometer in Jülich. Textures and Microstructures 8 \& 9, 457-466.

Schäfer, W., Jansen, E., Elf, F. and Will, G. (1984). A New Linear PositionSensitive Scintillation Detector for Neutron Powder Diffractometry. J. Appl. Cryst. 17, 159-166.

Wenk, H. R., Bunge, H. J., Jansen, E. and Pannetier, J. (1986). Preferred orientation of plagioclase-Neutron diffraction and U-stage data. Tectonophysics 126, 271-284.

Wenk, H. R., Kern, H., Schäfer, W. and Will, G. (1984). Comparison of neutron and X-ray diffraction in texture analysis of deformed carbonate rocks J. Struct. Geology 6, 687-692.

Will, G. (1979). POWLS: A powder least squares program J. Appl. Cryst. 12, 483-485.

Will, G., Höfler, S. \& Schäfer, W. (1986). Application of neutron diffraction in texture analysis Physica 136B, 473-476.

Will, G., Jansen, E. and Schäfer, W. (1983). POWLS-80. A programm for Refinement of Powder Diffraction Data. KFA-Report Jül-1987.

Will, G., Parrish, W. and Huang, T. C. (1983). Crystal-Structure Refinement by Profile Fitting and Least-Squares Analysis of Powder Diffractometer Data. J. Appl. Cryst. 16, 611-622. 